\title{
About the decision regularized equations of perturbed motion body
}

\author{
Vera Petelina, ${ }^{1, *}$ \\ ${ }^{1}$ Moscow State University of Civil Engineering, Yaroslavskoye shosse, 26, Moscow, 129337, Russia
}

\begin{abstract}
The article deals with determination of the second- and higherorder perturbations in Cartesian coordinates and body motion velocity constituents. A special perturbed motion differential equations system is constructed. The right-hand sides of this system are finite polynomials relative to an independent regularizing variable. This allows constructing a single algorithm to determine the second and higher order perturbations in the form of finite polynomials relative to some regularizing variables that are chosen at each approximation step. Following the calculations results with the use of the developed method, the coefficients of approximating polynomials representing rectangular coordinates and components of the regularized body speed were obtained. Comparison with the results of numerical integration of the equations of disturbed motion shows close agreement of the results. The developed methods make it possible to calculate, by the approximating polynomials, any intermediate point of the motion trajectory of the body.
\end{abstract}

\section{Introduction}

One of the crucial tasks associated with trajectory measurements is the determination of the partial derivatives of rectangular coordinates that make up the body motion speed with respect to the initial conditions. In operations [1-2] added auxiliary functions, which are degree series with respect to the auxiliary variable. In operations [3-5] outlined ways of using universal variables in a number of tasks of mechanics to determine disturbances by the method of variation of arbitrary constants. In this case, it is convenient to consider the components of the initial values of the radius-vector and velocity as osculating variables. New methods for determining disturbances keep the standard features of the classical ones, while calculating the disturbances, the small parameter method is used, which makes it possible to obtain asymptotic decomposition of the solution. Recently, Picard method for integration of differential equations is more commonly used, which leads to a convergent process of successive approximations that gives a solution to a system of differential equations.

The error of the solution depends on the accuracy of the initial approximation of the perturbation function. General principles of the development of perturbation theory in

\footnotetext{
* Corresponding author: verapetelina51@gmail.com
} 
coordinates were analyzed in operations [6-8] studied the use of regularizing variables for calculation of trajectories of motion. in operation. The results of this research show that the use of regularizing variables increases the computer-based accuracy of calculations and significantly reduces the calculation time.

A crucial task of mechanics is to approximate the rectangular coordinates that make up the body speed and time in case of disturbed motion by algebraic polynomials of the lowest degree with respect to the auxiliary variable with a predetermined degree of accuracy.

One of the important problems in mechanics is the approximation of rectangular coordinates constituting the body velocity and time when the motion is perturbed by the lowest degree algebraic polynomials relative to the auxiliary variable with a predetermined degree of accuracy.

This paper describes a special system of differential equations of the perturbed moving body and this system is integrated through successive approximations method, which using the coordinates and constituents body velocity, take the form of polynomials in powers of some auxiliary variable. Its own independent variable is taken at each approximation step.

\section{Mathematical model}

In works [9-10] there were found the equations which integrate Cartesian coordinates, constituents of regularized velocity and time with auxiliary variables $C_{i}$ as follows:

$$
\begin{gathered}
x=x_{0}+\sum_{i=1}^{7} C_{i} \cdot q_{1}^{(i)} x^{\prime}=x_{0}{ }^{\prime}+\sum_{i=1}^{7} C_{i} \cdot q_{4}^{(i)} \\
y=y_{0}+\sum_{i=1}^{7} C_{i} \cdot q_{2}^{(i)} y^{\prime}=y_{0}^{\prime}+\sum_{i=1}^{7} C_{i} \cdot q_{5}^{(i)} \\
z=z_{0}+\sum_{i=1}^{7} C_{i} \cdot q_{3}^{(i)} z^{\prime}=z_{0}^{\prime}+\sum_{i=1}^{7} C_{i} \cdot q_{6}^{(i)} \\
t=t_{0}+\sum_{i=1}^{7} C_{i} \cdot q_{7}^{(i)},
\end{gathered}
$$

where $q_{i}^{(k)}(i, k=1, \ldots, 7)$ - - is partial solutions of equiations in variations of regularized equations of a problem with 2 bodies. In order to find partial solutions $q_{i}^{(k)}(i, k=1, \ldots, 7)$ it is necessary to differentiate the general solution of regularized equations of an unperturbed problem with 2 bodies, by initial data $\xi_{0}, \eta_{0}, \zeta_{0}, \xi_{0}{ }^{\prime}, \eta_{0}{ }^{\prime}, \zeta_{0}{ }^{\prime}, t_{00}$.

In work [11] the following equations for defining first degree perturbations in values $C_{i}$ were found

$$
C_{i}=\frac{\mu_{1}}{\mu} \cdot \frac{\rho_{0}^{2}}{a_{1}^{2}} \cdot \int_{-1}^{u}\left[\frac{a_{1}^{3}}{\Delta_{1}^{3}} \cdot\left(E_{i x}^{*} \cdot \frac{x_{1}-x_{0}}{a_{1}}+E_{i y}^{*} \cdot \frac{y_{1}-y_{0}}{a_{1}}\right)+\frac{a_{1}^{3}}{r_{1}^{3}} \cdot\left(-E_{i x}^{*} \cdot \frac{x_{1}}{a_{1}}-E_{i y}^{*} \cdot \frac{y_{1}}{a_{1}}\right)\right] \cdot \frac{d \beta}{d u} \cdot d u,
$$

$(i=1,2,4,5,7)$

$$
C_{i}=\frac{\mu_{1}}{\mu} \cdot \frac{\rho_{0}^{2}}{a_{1}^{2}} \cdot \int_{-1}^{u}\left[\frac{a_{1}^{3}}{\Delta_{1}^{3}} \cdot E_{i z}^{*} \cdot \frac{z_{1}-z_{0}}{a_{1}}+\frac{a_{1}^{3}}{r_{1}^{3}} \cdot\left(-E_{i z}^{*}\right) \cdot \frac{z_{1}}{a_{1}}\right] \cdot \frac{d \beta}{d u} \cdot d u
$$


where $E_{i x}^{*}, E_{i y}^{*}, E_{i z}^{*}$ - is auxiliary variables which represent polynomials by degrees of regularized variable $\beta$

$$
\begin{aligned}
& E_{1 x}^{*}=\beta \quad E_{4 y}^{*}=\frac{\sqrt{2}}{3} \cdot \beta^{3} \\
& E_{1 y}^{*}=-\sqrt{2} \\
& E_{5 x}^{*}=\sqrt{2} \cdot\left(-\beta-\frac{1}{2} \beta^{3}\right) \\
& E_{2 x}^{*}=\sqrt{2} \cdot\left(1+\frac{3}{2} \beta^{2}\right) \quad E_{5 y}^{*}=1-\frac{1}{4} \beta^{4} \\
& E_{2 y}^{*}=-\beta+\frac{1}{2} \beta^{3} \quad E_{6 z}^{*}=1-\frac{1}{4} \beta^{4} \\
& E_{3 z}^{*}=-\beta-\frac{1}{2} \beta^{3} \quad E_{7 x}^{*}=\frac{1}{2} \beta^{2}+\frac{1}{3} \beta^{4}-\frac{1}{40} \beta^{6} \\
& E_{4 x}^{*}=1+\beta^{2}-\frac{\beta^{4}}{12} \quad E_{4 y}^{*}=\sqrt{2}\left(\frac{1}{6} \beta^{3}+\frac{3}{20} \beta^{5}\right)
\end{aligned}
$$

$x_{1}, y_{1}, z_{1}$ - coordinates of a perturbing body, $x_{0}, y_{0}, z_{0}$ - coordinates of the body studied in unperturbed motion.

Thus, expression for values $C_{i}$ may be represented as follows

$(\mathrm{i}=1,2,4,5,7)$

$$
C_{i}=\int_{-1}^{u} \frac{S_{i}(u)}{(1-u)^{2}} d u
$$

where,

$$
\begin{gathered}
S_{i}(u)=\frac{\mu_{1}}{\mu} \cdot \frac{\rho_{0}^{2}}{a_{1}^{2}} \cdot\left[\frac{a_{1}^{3} \cdot G_{1}(u)}{1-u} \cdot\left(E_{i x}^{*} \cdot \frac{x_{1}-x_{0}}{a_{1}}+E_{i y}^{*} \cdot \frac{y_{1}-y_{0}}{a_{1}}\right)+\frac{a_{1}^{3}}{r_{1}^{3}} \cdot\left(-E_{i x}^{*} \cdot \frac{x_{1}}{a_{1}}-E_{i y}^{*} \cdot \frac{y_{1}}{a_{1}}\right)\right] \cdot \frac{d \beta}{d u} \\
\frac{d \beta}{d u}=\frac{1}{2} \beta_{1} .
\end{gathered}
$$

Functions $E_{i x}^{*}, E_{i y}^{*}$ may be represented with the help of $\beta=\frac{1}{2} \cdot \beta_{1} \cdot(1+u)$, as polynomials by degrees of standardized regularizing variable $u$, whereas at $u=1, \beta=\beta_{1}, \beta_{1}=\sqrt{2 \cdot \frac{r}{\rho_{0}}-1}$ Coefficient $\beta_{1}$ corresponds to the moment of collision of the body being studied with the perturbing body. Then, functions $S_{i}(u)$ are shown as polynomials by degrees $u$ as follows

$$
S_{i}(u)=\sum_{k=0}^{n_{5}} S_{i k} \cdot u^{k}+\varepsilon\left(S_{i}, n_{5}\right)
$$

and functions $C_{i}(u)$ for trajectories of hitting into perturbing body look as follows 


$$
C_{i}(u)=\int_{-1}^{u} \frac{S_{i 0}}{(u-1)^{2}} d u+\int_{-1}^{u} \frac{S_{i 1} \cdot u}{(u-1)^{2}} d u+\ldots+\int_{-1}^{u} \frac{S_{i k} \cdot u^{k}}{(u-1)^{2}} d u+\ldots
$$

$(i=1,2,4,5,7)$

Let us enter a new regularizing variable:

$$
w=1-u
$$

Then, expressions (8) will have the following form:

$$
C_{i}(1-w)=-\int_{2}^{w} \frac{S_{i 0} \cdot d w}{w^{2}}-\int_{2}^{w} \frac{S_{i 1} \cdot(1-w) \cdot d w}{w^{2}}-\cdots-\int_{2}^{w} \frac{S_{i k} \cdot(1-w)^{k} \cdot d w}{w^{2}}-\cdots
$$

Integrals are easy to calculate:

$$
\begin{aligned}
& -\int_{2}^{w} \frac{S_{i 0} \cdot d w}{w^{2}}=S_{i 0} \cdot\left(\frac{1}{w}-\frac{1}{2}\right) \\
& -\int_{2}^{w} \frac{S_{i 1} \cdot(1-w) \cdot d w}{w^{2}}=S_{i 1} \cdot\left(\frac{1}{w}+\ln w-\frac{1}{2}-\ln 2\right) \\
& \quad-\int_{2}^{w} \frac{S_{i 2} \cdot(1-w)^{2} \cdot d w}{w^{2}}=S_{i 2} \cdot\left(\frac{1}{w}+2 \ln w-w+\frac{3}{2}-2 \ln 2\right) \\
& -\int_{2}^{w} \frac{S_{i 3} \cdot(1-w)^{3} \cdot d w}{w^{2}}=S_{i 3} \cdot\left(\frac{1}{w}+3 \ln w-3 w+\frac{1}{2} w^{2}+\frac{7}{2}-3 \ln 2\right) \\
& -\int_{2}^{w} \frac{S_{i 4} \cdot(1-w)^{4} d w}{w^{2}}=S_{i 4} \cdot\left(\frac{1}{w}+4 \ln w-6 w+2 w^{2}-\frac{1}{3} w^{3}+\frac{37}{6}-4 \ln 2\right) \\
& -\int_{2}^{w} \frac{S_{i 5} \cdot(1-w)^{5} d w}{w^{2}}=S_{i 5} \cdot\left(\frac{1}{w}+5 \ln w-10 w+5 w^{2}-\frac{5}{3} w^{3}+\frac{w^{4}}{4}+\frac{53}{6}-5 \ln 2\right) \\
& C_{i}(1-w)=\tilde{C}_{i}(w),
\end{aligned}
$$

$(i=1,2,4,5,7)$

Thus, first-degree perturbations in auxiliary coefficients $\tilde{C}_{i}$ have the form:

$$
\tilde{C}_{i}(w)=\frac{P_{i 0}}{w}+P_{i 1} \cdot \ln w+Q_{i}(w)+\varepsilon\left(Q_{i}\right),
$$

where:

$$
Q_{i}(w)=\sum_{k=0}^{n_{6}} Q_{i k} \cdot w^{k}+\varepsilon\left(Q_{i}, n_{6}\right)
$$


$(i=1,2,4,5,7)$

Particular solutions for equations in variations of regularized equations of a problem with 2 bodies, are shown in work [11] as polynomials by degrees of regularizing variable $\beta$.By putting expression $\beta=\frac{1}{2} \cdot \beta_{1} \cdot(1+u)$, into expressions $q_{i}^{(k)}(\beta)$, receive particular solutions $q_{i}^{(k)}$ as polynomials by degrees of regularizing variable $u$; then, by putting expressions (9) into obtained expressions $q_{i}^{(k)}$, receive particular solutions $q_{i}^{(k)}$ as polynomials by degrees of regularizing variable $w$. By a similar substitution, receive unperturbed-motion polynomials $x_{0}, y_{0}, x_{0}^{\prime}, y_{0}^{\prime}, t_{0}$ by degrees of variable By putting the obtained expressions into formulae (1) receive rectangular coordinates $x, y$, constituents of regularized velocity $x^{\prime}, y^{\prime}$, time $t$ in the 1 rst approximation as follows

$$
\begin{gathered}
x=\frac{A_{1}}{w}+R_{x}(w) \cdot \ln w+R_{x 1}(w)+\varepsilon\left(R_{x 1}, n^{*}\right) \\
y=\frac{A_{2}}{w}+R_{y}(w) \cdot \ln w+R_{y 1}(w)+\varepsilon\left(R_{y 1}, n^{*}\right) \\
x^{\prime}=\frac{A_{3}}{w}+R_{x^{\prime}}(w) \cdot \ln w+R_{x^{\prime} 1}(w)+\varepsilon\left(R_{x^{\prime} 1}, n^{*}\right) \\
y^{\prime}=\frac{A_{4}}{w}+R_{y^{\prime}}(w) \cdot \ln w+R_{y^{\prime} 1}(w)+\varepsilon\left(R_{y^{\prime} 1}, n^{*}\right) \\
t=\frac{A_{5}}{w}+R_{t}(w) \cdot \ln w+R_{t 1}(w)+\varepsilon\left(R_{t 1}, n^{*}\right),
\end{gathered}
$$

where $R_{x}, R_{x 1}, R_{y}, R_{y 1}, R_{x^{\prime}}, R_{x^{\prime} 1}, R_{y^{\prime}}, R_{y^{\prime} 1}, R_{t}, R_{t 1}$ - polynomials by degrees $w$, whereas, errors may be done arbitrary small at selection of a respective value $n^{*}$. In expressions (14) for rectangular coordinates, constituents of regularized velocity and time of perturbed motion of spacecraft, there are available terms at $\frac{1}{w}$ and $\ln w$. Enter a new regularizing variable $v_{1}$ as follows:

$$
\begin{gathered}
\ln w=\alpha_{1} \cdot v_{1}+\beta_{1} \\
w=e^{\alpha_{1} v_{1}+\beta_{1}}=e^{\beta_{1}} \cdot\left(1+\frac{\alpha_{1} v_{1}}{1 !}+\frac{\alpha_{1}^{2} v_{1}^{2}}{2 !}+\cdots+\frac{\alpha_{1}^{n} v_{1}^{n}}{n !}+\cdots+\right) \\
w^{-1}=e^{-\alpha_{1} v_{1}-\beta_{1}}=e^{-\beta_{1}} \cdot\left(1-\frac{\alpha_{1} v_{1}}{1 !}+\frac{\alpha_{1}^{2} v_{1}^{2}}{2 !}-\frac{\alpha_{1}^{3} v_{1}^{3}}{3 !}+\cdots+\frac{(-1)^{n} \alpha_{1}^{n} v_{1}^{n}}{n !}+\cdots\right)
\end{gathered}
$$

Thus, substituting relations (15) and (16) into expressions (14), we have that rectangular coordinates $x, y, z$, constituents of regularized velocity $x^{\prime}, y^{\prime}, z^{\prime}$, time $t$ are integral functions of a new regularizing variable $v_{1}$, which enables to present 
the specified functions by terminating polynomials with the accuracy degree preset in advance.

Let us define values $\alpha_{1}$ and $\beta_{1}$ from boundary conditions, which have the look at $u=u^{*}, v_{1}=1$, at $u=-1, v_{1}=-1$. The value of regularizing variable $u^{*}$ or respectively $w^{*}$ correlates with the moment of the body studied getting into the effective area of the perturbing body and $u^{*} \prec 1, w^{*} \prec 1$. We have

$$
\left\{\begin{array}{l}
1-u^{*}=e^{\alpha_{1}+\beta_{1}} \\
2=e^{\beta_{1}-\alpha_{1}}
\end{array}\right.
$$

Where as $\quad \alpha_{1} \prec \beta_{1}$.

Thus, by carrying out a number of substitutions of the independent first-degree perturbation variable in rectangular coordinates, constituents of regularized velocity and time of motion, bodies are represented as terminating polynomials of comparatively low degree and relatively regularizing variable with a sufficiently high accuracy degree.

Functional diagram of calculation of first-order perturbations by analytical method, consists of the following main blocks. The first block is concluded in building-up polynomials which show coordinates of the perturbing body, coordinates, constituents of regularized velocity and time of unperturbed motion of the body studied by degrees of standardized regularizing variable $u$. Calculation results demonstrate coefficients of respective polynomials.

The second block consists in representing the mutual distance square between the body studied and the perturbing body $\Delta_{1}^{2}$ as polynomial by degrees of independent regularizing variable $u$.

In the third block of the programme, finding of roots of equation $\Delta_{1}^{2}=0$ is carried out with the help of iterations, i.e. determination of those values of the independent regularizing variable $u$, at which collision of the body studied with the perturbing body takes place and which we will call critical points. Numerical analysis of the closest pair of critical points in the vicinity of the main interval of variation of regularizing variable $u$ is carried out; the analysis demostrated that for trajectories of hitting into perturbing body, point $u=1$ is such critical point, at which the distance from the body studied to perturbing body turns to zero, the remaining pairs of critical points which are complex-conjugate, are at significant distances. Polynomials are built-up,

$$
F_{1}(u)=\frac{\Delta_{1}(u)}{(u-1)^{2}} \quad G_{1}(u)=\frac{1}{\left[F_{1}(u)\right]^{3 / 2}}
$$

by degrees of regularized variable $u$. Results represent coefficients of respective polynomials. 
Block four of the programme consists in identifying the main part of perturbations in auxiliary variables $C_{i}$ in the form of expansion as functions $S_{i}(u)$

$$
\begin{gathered}
C_{i}(u)=\int_{-1}^{u} \frac{S_{i k} \cdot u^{k} \cdot d u}{(u-1)^{2}} \\
C_{i}^{*}=\frac{\mu_{1} \cdot \rho_{0}}{\mu} \cdot \int_{-1}^{u} \frac{a_{1} \cdot G_{1}(u)}{1-u} \cdot\left(E_{i x}^{*} \cdot \frac{x_{1}-x_{0}}{a_{1}}+E_{i j}^{*} \cdot \frac{y_{1}-y_{0}}{a_{1}}\right) \cdot \frac{d \beta}{d u} \cdot d u,
\end{gathered}
$$

$$
(i=1,2,4,5,7)
$$

Determination of auxiliary functions $E_{i x}^{*}$ and $E_{i y}^{*}$ is carried out in the form of polynomials by degrees of regularized variable $u$. Functions $E_{i x}^{*}$ and $E_{i y}^{*}$ are dimensionless factors with respect to $D_{i k} / D$, where $D$ - is determinant of fundamental matrix of equation partial solutions in variations of regularized equations of a problem with 2 bodies [5], $D_{i k}$ - respective algebraic complements of the partial solution matrix. Entering of a new regularizing variable $w$ and determination of the main part of perturbations in auxiliary variables $C_{i}$ in polynomial- transcendent form with respect to regularizing variable $w$ is performed. Programme's block five represents determination of indirect part of perturbations in auxiliary variables $C_{i}$

$$
C_{i}^{* *}=\frac{\mu_{1} \cdot \rho_{0}^{2}}{\mu} \cdot \int_{-1}^{u} \frac{a_{1}}{r_{1}^{3}} \cdot\left(-E_{i x}^{*} \cdot \frac{x_{1}}{a_{1}}-E_{i y}^{*} \frac{y_{1}}{a_{1}}\right) \cdot \frac{d \beta}{d u} \cdot d u,
$$

$$
(i=1,2,4,5,7)
$$

as polynomials by degrees of regularizing variable $w$ and determination of general perturbations in auxiliary variables $C_{i}$ in polynomial- transcendent form with respect to variable $w$.

Programme's block six consists in determination of partial solutions of $q_{i}^{(k)}$ equations in variations of regularized equations of problem of 2 bodies in the form of polynomials by degrees of regularized variable $w$ as well as in determination of rectangular coordinates, constituents of regularized velocity and time of perturbed motion of the body studied in a first approximation in polynomial- transcendent form with respect to independent regularizing variable $w$. Calculation results represent coefficients of respective polynomials.

Programme's block seven consists in determination of rectangular coordinates, constituents of regularized velocity and time of perturbed motion of the body studied in the form of polynomials by degrees of a new regularizing variable $v_{1}$ which is entered with the help of relation (15). 


\section{Conclusions}

Thus, by carrying out a number of substitutions of the independent first-degree perturbation variable in rectangular coordinates, constituents of regularized velocity and time of motion, bodies are represented as terminating polynomials of comparatively low degree with respect to regularizing variable with a sufficiently high accuracy degree. At each phase of calculations, a reduction in degree of respective polynomials with the help of Chebyshev polynomials and control of computation results by the initial and final point were carried out. Additionally, standardization of regularizing variable ${ }^{w}$ for the purpose of reducing the degree of polynomials which represent the required functions with the preset accuracy degree on interval $(-1 ; 1)$ was conducted.

The suggested method may be used at simulating the deformation of metal ridged outer shell-plating of panels and interior shell plating made of aluminium foil. At developing the procedure for calculation of multi-layer construction structures which are subjected to the impact of shock-waves, as well in studying construction structures, in particular, cross-bending of cantilevers, at simulating tensions and deformations in construction elements effected by static loads of various value and configuration. As well, the procedure may be used at calculation of the structure in terms of seismic effect.

\section{References}

1. P. Xu, Comm. In Nonl. Sci. and Num. Sim., 59, 515-543 (2018) doi:10.1016/j.cnsns.2017.11.021

2. K. Schiefermayr, Jour. of Math. Analysis and App., 445, 871-883 (2017) doi:10.1016/j.jmaa.2016.08.021

3. Z. Fuqiang, H. Qingxue, X. Jiaquan, L. Yugui, M. Lifeng, W. Jianmei, App. Math. and Comp., 313, 321-330 (2017)

4. V. Lychagin, V. Yumaguzhin, Jour. of Geom. and Phys., 130, 213-228 (2018) doi:10.1016/j.geomphys.2018.03.025

5. Z. Wen, G. Gundersen, J. Heittokangas, Jour. of Differ. Equa., 264, 98-114 (2018) doi:10.1016/j.jde.2017.09.003

6. H. Tong, M. Ng, Jour. of Comp., 49, 85-94, (2018) doi.org/10.1016/j.jco.2018.08.001

7. N. Huu Can, N. Huy Tuan, V. Van Au, L. Duc Thang, Jour. of Math. Analysis and App., 462, 1148-1177 (2018) doi.org/10.1016/j.jmaa.2018.01.066

8. D. Torre, R.Flores, E.Fantino, Acta Astronautica, 153, 26-38 (2018) doi:10.1016/j.actaastro.2018.10.010

9. V. Petelina, MATEC, 117, 00135 (2017) doi:10.1051/matecconf $/ 201711700135$

10. V. Petelina, MATEC, 196, 01016 (2018) doi:10.1051/matecconf $/ 201819601016$

11. V. Petelina, IOP Conf. Ser: Mater., 456, 012123 (2018) doi:10.1088/1757$899 \mathrm{X} / 456 / 1 / 012123$ 